\title{
Nasal nitric oxide and nitric oxide synthase expression in primary ciliary dyskinesia
}

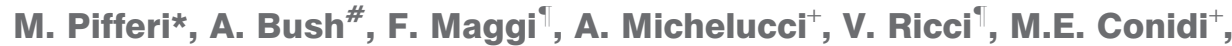 \\ A.M. Cangiotti ${ }^{\S}$, A. Bodini ${ }^{f}$, P. Simi ${ }^{+}$, P. Macchia* and A.L. Boner ${ }^{f}$
}

ABSTRACT: No study has evaluated the correlation between different expression of nitric oxide synthase (NOS) isoforms in nasal epithelial cells and nasal NO (nNO) level in primary ciliary dyskinesia (PCD).

Gene expression of endothelial (NOS3) and inducible NOS (NOS2) and their correlation with nNO level, ciliary function and morphology were studied in patients with PCD or secondary ciliary dyskinesia (SCD). NOS3 gene polymorphisms were studied in blood leukocytes.

A total of 212 subjects were studied (48 with PCD, 161 with SCD and three normal subjects). nNO level correlated with mean ciliary beat frequency $(p=0.044 ; r=0.174)$. The lower the nNO level the higher was the percentage of immotile cilia $(p<0.001 ; r=-0.375)$. A significant positive correlation between NOS2 gene expression and nNO levels was demonstrated in all children $(p=0.001 ; r=0.428)$, and this correlation was confirmed in patients with $P C D(p=0.019 ; r=0.484)$. NOS2 gene expression was lower in PCD than in SCD $(p=0.04)$. The NOS3 isoform correlated with missing central microtubules $(p=0.048 ; r=0.447)$. $n N O$ levels were higher in PCD subjects with the NOS3 thymidine 894 mutation, and this was associated with a higher ciliary beat frequency $(p=0.045)$.

These results demonstrate a relationship between nNO level, NOS mRNA expression and ciliary beat frequency.

KEYWORDS: Gene polymorphism, nasal nitric oxide, nitric oxide synthase, primary ciliary dyskinesia, secondary ciliary dyskinesia

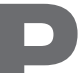

rimary ciliary dyskinesia (PCD; Mendelian Inheritance in Man (MIM) identifier 244400) is a rare (one per 15,000-30,000 live births) and usually autosomal recessive disease associated with situs inversus in nearly a half of cases [1]. Impaired mucociliary clearance due to defective motility of cilia is the hallmark of the condition, but an additional sensitive, although not specific, feature of PCD is the very low level of nasal nitric oxide (nNO). For this reason, $\mathrm{nNO}$ level has been proposed as a screening tool for PCD [1], even though the mechanism of low $\mathrm{nNO}$ levels in PCD is not known [2-4].

$\mathrm{NO}$ is a product of L-arginine metabolism by one of three isoforms of nitric oxide synthase (NOS): endothelial (NOS3, located on chromosome 7), neuronal (NOS1, located on chromosome 12), and inducible (NOS2, located on chromosome 17). At least in asthmatics, it is believed that NOS2 is the major source of exhaled NO. NOS2 is localised close to cilia in the apical part of nasal epithelial cells [5]. It has been speculated that, in PCD, there is either uncoupling of the contractile process of the cilia from NOS, leading to failure of $\mathrm{NO}$ production, or that a low nNO level is an expression of a generalised disorder of airway NO metabolism, with abnormalities in all three NOS isoforms [6].

It has been established that all three NOS isoforms may be regulated by gene induction at multiple levels, including transcriptional control [7]. The NOS3 gene, which is located in chromosomal region 7q35-36, contains a number of variants, including a guanine $(\mathrm{G}) /$ thymidine $(\mathrm{T})$ polymorphism at position 894 in exon 7 [8]. This $\mathrm{G}$ to $\mathrm{T}$ substitution at nucleotide $894(894 \mathrm{G}>\mathrm{T})$, which results in an aspartate rather than a glutamate at position 298 in NOS3, is of potential functional relevance since it was shown to correlate with severity of cardiopulmonary diseases in non-PCD patients [9], including those with asthma and cystic fibrosis [10, 11], but no data are available in PCD.

Since the low nNO level in PCD may be related to altered expression of NOS isoforms, we hypothesised that, in PCD, there would be a reduction in NOS2 and NOS3 mRNA expression in the nasal
AFFILIATIONS

Depts of *Pediatrics

-Experimental Pathology, and

${ }^{+}$Cytogenetics and Molecular Genetic

Unit, University of Pisa, Pisa,

${ }^{\S}$ Electron Microscopy Unit, Institute

of Normal Human Morphology,

Umberto I Hospital, Marche

Polytechnic University, Ancona,

${ }^{f}$ Dept of Pediatrics, University of

Verona, Verona, Italy.

\#Imperial School of Medicine at the

National Heart and Lung Institute,

London, UK.

CORRESPONDENCE

M. Pifferi

Dept of Pediatrics

University of Pisa

Via Roma 67

56126 Pisa

Italy

E-mail: m.pifferi@med.unipi.it

Received:

March 212010

Accepted after revision:

Oct 112010

European Respiratory Journal

Print ISSN 0903-1936

Online ISSN 1399-3003 
mucosa, compared with a comparably infected and inflamed control group who did not have a congenital defect of ciliary function, namely children with secondary ciliary dyskinesia (SCD). The aim of the present study was to determine whether NOS2 and NOS3 gene expression are different in nasal ciliate cells of children with PCD and SCD, respectively, and whether gene expression levels correlate with $\mathrm{nNO}$ levels. SCD patients showed acquired ciliary abnormalities caused by viral or bacterial respiratory infections, and irritant injury of the mucosa produced by air pollution.

NOS3 was studied since it is the only NOS isoform known to be localised in normal human nasal mucosa and is mainly associated with the ciliary basal microtubule membrane [5]. The relationship of NOS3 gene expression to ultrastructural abnormalities of cilia was assessed. Furthermore, the relationship of the $894 \mathrm{G}>\mathrm{T}$ polymorphism to NOS3 gene expression and $\mathrm{nNO}$ levels was analysed in PCD patients, since it had been previously demonstrated that variants in the genes encoding the NOSs may act as disease modifier loci in cystic fibrosis [9]. Finally, it was assessed whether the 894G $>$ T polymorphism in the NOS3 gene was associated with particular ultrastructural and functional abnormalities of cilia.

\section{MATERIALS AND METHODS}

\section{Subjects}

Informed consent for all procedures was obtained from the children's parents, and the Hospital Ethical Committee of Pisa Hospital (Pisa, Italy) approved the protocol. Between January 2006 and June 2009, 212 children and adolescents (105 male and 107 female) aged 1 month to 17.5 yrs (median 6.2 yrs; interquartile range (IQR) 5.1 yrs) with a clinical history and signs and symptoms suggestive of PCD were consecutively evaluated in the Dept of Pediatrics (University of Pisa, Pisa, Italy), a national referral centre. Investigations in all of the children included serum immunoglobulins (Igs), including total and specific IgE and IgG subclasses, lymphocytic subsets, the sweat test and cystic fibrosis gene mutations. Young children were carefully observed during feeding in order to exclude swallowing problems and possible aspiration. Careful examination of the upper airway was performed to exclude clinical signs of supra-oesophageal complications of gastrooesophageal reflux in all of the patients. Sputum or a cough swab was collected for microbiological analysis in subjects able to cooperate. In children too young to cooperate, a pharyngeal aspirate specimen was obtained after an overnight fast using a disposable catheter connected to a mucus extractor inserted into the mouth to a depth of 7-10 cm and drawn back while applying gentle suction.

Evaluations for PCD were performed when subjects had been free from acute respiratory infection for $\geqslant 4$ weeks and, in order to reduce the risk of infections causing secondary ciliary abnormalities, the patients were treated with oral antibiotics throughout this period. Patients were required to stop any pharmacological treatment except antibiotics in the previous $48 \mathrm{~h}$. Diagnosis of PCD and SCD was made on the basis of structural and/or functional ciliary abnormalities. In all subjects, ciliary motion analysis, ultrastructural assessment of cilia on nasal brushings and $\mathrm{nNO}$ measurement were performed.
nNO measurements, nasal brush biopsy, ciliary motion analysis and ultrastructural studies were performed according to standard methodologies [12-16], detailed in the online supplementary material.

\section{Measurement of NOS2 and NOS3 mRNA}

NOS2 and NOS3 were evaluated in random samples of 71 and 53 children, respectively (randomisation number generated by an online program [17]). Nasal ciliate cells were immediately centrifuged and the cell pellets obtained were repeatedly washed in abundant PBS, resuspended in $400 \mu \mathrm{L}$ PBS and finally stored at $-80^{\circ} \mathrm{C}$ until use. Total RNA from ciliate cells extracted using the RNeasy Mini Kit (QIAgen, Frederick, MD, USA) was reverse transcribed to cDNA (Fermentas, Glen Burnie, MD, USA). NOS2 and NOS3 mRNA was measured using a commercial real-time quantitative PCR performed on an ABI PRISM 7700 (Applied Biosystems, Foster City, MA, USA) with primers and probes specific for each gene (TaqMan Gene Expression assays; Applied Biosystems). cDNA were also used as templates for reduced glyceraldehye-3-phosphate dehydrogenase (GAPDH) quantification. Each sample was amplified in triplicate and a negative PCR control, without cDNA template, was included in each assay. The relative expression ratio $(R)$ of the targets (NOS2 and NOS3 genes) was computed using the Relative Expression Software Tool [18], which permits relative quantification between groups and a normalisation of the target genes with a reference gene. This software calculates the $R$ of a target gene on the basis on its real-time PCR efficiency $(E)$ and the crossing point $(\mathrm{Cp})$ difference $(\Delta)$ of an unknown sample versus a control called calibrator. In the present study, the calibrator was randomly selected by the principal investigator (M. Pifferi) from among three children with normal ciliary motion and normal transmission electron microscopy (TEM) results. The target gene expression was normalised by GAPDH, a nonregulated reference gene expression, using the formula:

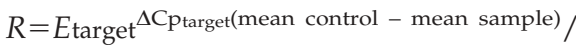

$$
\begin{aligned}
& E_{\text {reference }} \Delta \mathrm{C} \text { preference(mean control - mean sample) }
\end{aligned}
$$

\section{Genotyping}

In 48 PCD children, blood was drawn to determine whether or not the NOS3 gene polymorphism $894 \mathrm{G}>\mathrm{T}$ was present. Genomic DNA was extracted from peripheral blood leukocytes using the EZ1 DNA kit and BioRobot EZ1 (Qiagen). The presence of the $894 \mathrm{G}>\mathrm{T}$ polymorphism (single nucleotide polymorphism identifier rs1799983) in the NOS3 gene (Ensembl gene identifier ENSG00000164867) was examined by sequencing analysis.

The primers used for amplification were as follows: forward primer: 5'-GGCTGGACCCCAGGAAAC-3', and reverse primer: 5'-GCACCTCAAGGACCAGCTC-3'.

The PCR conditions were $5 \mathrm{~min}$ at $95^{\circ} \mathrm{C}$, followed by 30 cycles of $30 \mathrm{~s}$ at $94^{\circ} \mathrm{C}, 30 \mathrm{~s}$ at $60^{\circ} \mathrm{C}$ and $30 \mathrm{~s}$ at $72^{\circ} \mathrm{C}$, and a final elongation for $7 \mathrm{~min}$ at $72^{\circ} \mathrm{C}$.

Purified PCR products were sequenced, with the same forward primer as used for the amplification, according to the manufacturer's instructions and using the Big Dye Terminator v3.1 Cycle Sequencing Kit (Applied Biosystems) and ABI PRISM 3130xl Genetic Analyser (Applied Biosystems). 


\section{Statistical analyses}

Baseline variables were expressed as median and IQR, and differences between medians were assessed using the MannWhitney (Wilcoxon) W-test. Receiver operating characteristic curves were used to calculate the sensitivity and specificity of nNO level for the diagnosis of PCD, and positive and negative predictive values were generated using standard equations.

Correlations between the quantitative results of ciliary motion analysis, ultrastructural evaluations, NOS2 and NOS3 gene expression measurements, the presence or absence of the $894 \mathrm{G}>\mathrm{T}$ polymorphism (analysed as a binary variable: $\mathrm{GG}=0$; GT or TT=1) and nNO measurements were examined using Spearman's rank correlation test. The Chi-squared test was used to evaluate the association between the presence of the $894 \mathrm{~T}$ variant in the NOS3 gene and $\mathrm{nNO}$ levels $\geqslant 290 \mathrm{ppb}$ in PCD patients, and to assess the association between chronic airway infection with Pseudomonas aeruginosa and the presence of the $894 \mathrm{~T}$ allele.

A p-value of $<0.05$ was considered significant. All statistical calculations were performed using SPSS version 15.0 software for Windows (SPSS, Inc., Chicago, IL, USA) for personal computers.

\section{RESULTS}

No child had cystic fibrosis (negative sweat test and negative mutation analysis results), immunological abnormalities or, in young children, swallowing problems and clinical signs of gastro-oesophageal reflux. Ciliary motion analysis (abnormal motion patterns, including immotile cilia and/or a very low ciliary beat frequency (CBF)) and TEM evaluation of cilia (alterations of the central pair and dynein arms deficiencies, associated with a small proportion of swollen cilia and compound cilia) led to the diagnosis of PCD in $48(22.6 \%)$ out of 212 children, $32(66.7 \%)$ with situs inversus (table 1). Levels of $\mathrm{nNO}$ were $<290 \mathrm{ppb}$ in $38(79.2 \%)$ out of $48 \mathrm{PCD}$ subjects. In the remaining 10 patients with levels of $\mathrm{nNO}$ $\geqslant 290 \mathrm{ppb}$, the diagnosis of PCD was confirmed by ciliary activity evaluation following ciliogenesis in culture. In the present study group, the nNO cut-off level with the best combination of sensitivity (74\%) and specificity (80\%) was $290 \mathrm{ppb}$, which had a positive and negative predictive value of 76 and $78 \%$, respectively. Ciliary motion analysis demonstrated abnormal patterns in a small proportion of cilia, with some thick cilia and a low CBF, compatible with the diagnosis of SCD in $161(75.9 \%)$ patients. In these subjects, TEM showed nonspecific abnormalities compatible with chronic inflammation (prevalence of swollen cilia and compound cilia). Three subjects exhibited no abnormalities in TEM results and ciliary function, one of whom was randomly selected as a healthy control for NOS2 and NOS3 gene expression measurements.

Significant correlations were found between ciliary motion analysis and TEM results. In particular, taking all 212 subjects together, abnormal motion patterns (as a percentage of motile cilia) were directly correlated $(\mathrm{p}=0.04 ; \mathrm{r}=0.121)$ and mean $\mathrm{CBF}$ (immotile cilia excluded) was inversely correlated $(p<0.001$; $r=-0.346)$ with the percentage of pathological cilia observed using TEM. Mean CBF was directly correlated and immotile cilia inversely correlated with a normal ciliary axoneme on

\begin{tabular}{|c|c|c|}
\hline & PCD & SCD \\
\hline Subjects n (\%) & $48(22.6)$ & $161(75.9)$ \\
\hline \multicolumn{3}{|l|}{ nNO n (\%) } \\
\hline$<290$ ppb & $38(79.2)$ & $21(13)$ \\
\hline$\geqslant 290 \mathrm{ppb}$ & $10(20.8)$ & $140(87)$ \\
\hline \multicolumn{3}{|c|}{ NOS mRNA fold increase ${ }^{\#}$} \\
\hline NOS3 & $4.5(18.3)^{\bullet}$ & $7.4(15.8)^{+}$ \\
\hline NOS2 & $0.9(1.9)^{\circ}$ & $1.9(3.5)^{\S}$ \\
\hline \multicolumn{3}{|c|}{ NOS3 genotype n (\%) } \\
\hline TT & $4(8.3)$ & \\
\hline GT & $22(45.8)$ & \\
\hline GG & $22(45.8)$ & \\
\hline
\end{tabular}

Data are presented as median (interquartile range), unless otherwise indicated. PCD: primary ciliary dyskinesia; SCD: secondary ciliary dyskinesia; nNO: nasal nitric oxide; NOS: nitric oxide synthase; T: thymidine; G: guanine. ${ }^{\#}$ : versus normal control; "? in 27 patients; ${ }^{+}$: in 26 patients; ${ }^{\text {s. in }} 44$ patients.

ultrastructural evaluation $(\mathrm{p}<0.001 ; \mathrm{r}=0.346$ and $\mathrm{p}<0.001$; $r=-0.436$, respectively)

Considering PCD and SCD children together, nNO level was very weakly correlated with mean CBF $(p=0.044 ; r=0.174)$ (fig. 1) and inversely correlated with the percentage of immotile cilia on light microscopy ( $<<0.001 ; \mathrm{r}=-0.375)$ (fig. 2).

Of the 71 patients in whom NOS2 was evaluated, 27 had PCD and 44 SCD. NOS3 was evaluated in 53 patients (27 PCD and 26 SCD). Relative NOS2 gene expression in nasal ciliate cells of children with PCD (median 0.9 (IQR 1.9)-fold increase versus normal control) was significantly lower $(p=0.03)$ than that in subjects with SCD (1.9 (3.5)-fold increase versus normal control), although there was considerable overlap, i.e. SCD children showed greater NOS2 gene expression than PCD individuals or the normal control, the latter two not being significantly different. There was no difference in NOS3 gene

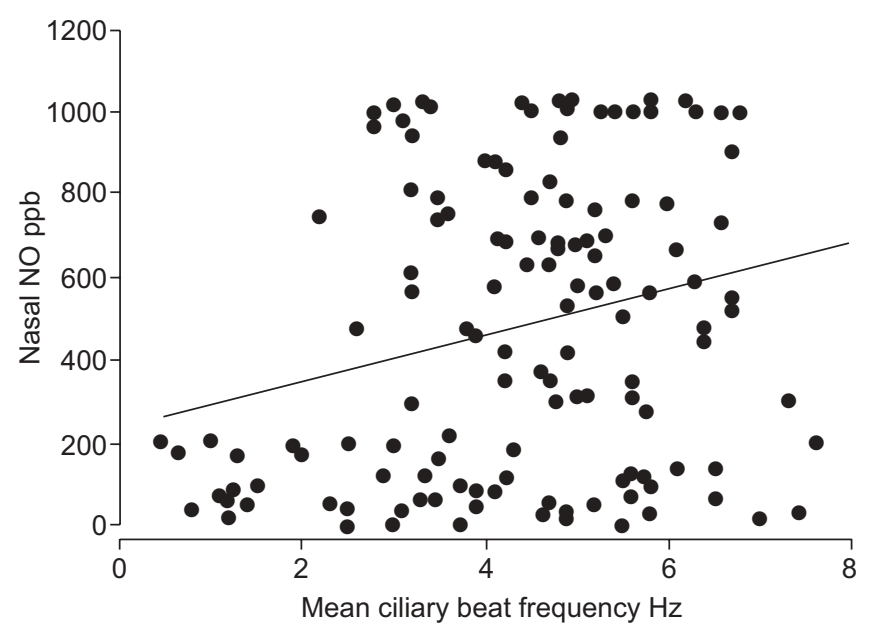

FIGURE 1. Correlation between nasal nitric oxide (NO) concentration and mean ciliary beat frequency $(p=0.044 ; r=0.174)$. 


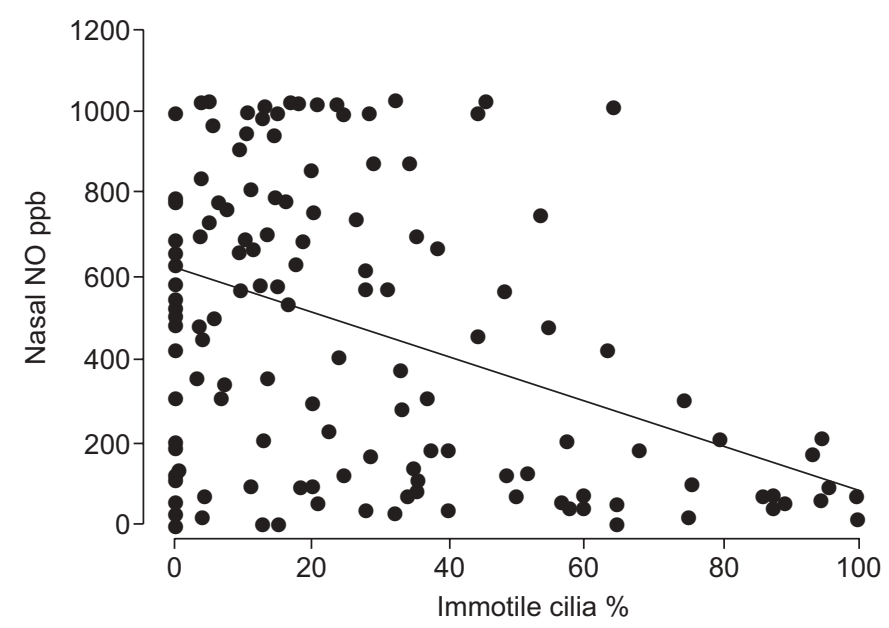

FIGURE 2. Correlation between nasal nitric oxide (NO) concentration and percentage of immotile cilia on light microscopy $(p<0.001 ; r=-0.375)$.

expression between children with PCD (4.5 (18.3)-fold increase compared with normal control) and subjects with SCD (7.4 (15.8)-fold increase compared with normal control). There was a significant correlation $(\mathrm{p}=0.019 ; \mathrm{r}=0.484)$ between NOS2 gene expression and nNO level in the 27 children with PCD, but not in SCD. However, the significance was lost if the three outliers were excluded. In any case, a significant positive correlation between NOS2 gene expression and $\mathrm{nNO}$ levels was demonstrated in all children (PCD plus SCD) ( $p=0.001$; $\mathrm{r}=0.428)$.

There was no correlation between relative NOS3 gene expression and $\mathrm{nNO}$ levels in any group. However, since the NOS3 isoform is localised at the basal membrane of ciliary microtubules, a significant correlation with ultrastructural alterations was sought and found, such as missing central microtubules in patients with PCD $(p=0.048 ; r=0.447)$, but not in subjects with SCD. This correlation disappeared when the extreme outlier with $>250 \%$ NOS3 gene expression relative to the healthy normal comparator was excluded. However, numbers were small, and there was a wide scatter about the line of best fit.

The NOS3 genotype was homozygous for the wild-type $894 \mathrm{G}$ allele in 22 patients; the $\mathrm{T}$ allele $(\mathrm{n}=26)$ was heterozygous (894GT) in 22 and homozygous (894TT) in four. Median (IQR) nNO concentration in GG wild-type subjects was not significantly different from that in children carrying the $\mathrm{T}$ allele (93.0 (132.3) versus 75.9 (326.9) ppb); however, the numbers were small.

nNO levels $\geqslant 290 \mathrm{ppb}$ were observed in $10 \mathrm{PCD}$ patients, seven (five of which were adolescent females) with the 894T mutant allele $(26.9 \%$ of the total with the $\mathrm{T}$ allele) and three homozygous for the $894 \mathrm{G}$ wild-type allele $(13.6 \%$ of the total number of subjects homozygous for the G allele).

No significant difference was found in NOS3 mRNA between PCD patients homozygous for the $894 \mathrm{G}$ allele and those carrying the $894 \mathrm{~T}$ allele in either the heterozygous or homozygous form (4.6 (17.7)- and 3.2 (7.5)-fold increase versus normal control, respectively).
Finally, the $\mathrm{T}$ allele (in either heterozygotes or homozygotes) inversely correlated with the most typical ultrastructural alterations, such as missing inner and outer dynein arms $(\mathrm{p}=0.002 ; \mathrm{r}=0.480)$, and this was not the case with the wildtype GG genotype. In addition, the inner and outer dynein arm defect was significantly reduced when $894 \mathrm{~T}$ allele was present (47.5 (59.3) and $0(2.6) \%$, respectively, in 894GG versus 894GT plus 894TT genotypes $(p=0.006))$. There was a significant difference in CBF between PCD patients carrying $\mathrm{T}$ variants and PCD patients homozygous for the $G$ allele $(p=0.0459)$.

Moreover, chronic infection with Pseudomonas aeruginosa was found in seven $(31.8 \%)$ out of 22 of the patients homozygous for the $\mathrm{G}$ allele and in five $(19.2 \%)$ out of 26 patients carrying the $\mathrm{T}$ variant $(\mathrm{p}=0.5)$. However, three out of six pubertal female patients homozygous for the $G$ allele were chronically infected with Pseudomonas aeruginosa, compared with none of five pubertal female patients carrying the $\mathrm{T}$ allele, but, again, numbers were small and so any conclusions must be provisional pending confirmation.

\section{DISCUSSION}

The main findings of the present study are that NOS2 mRNA levels correlated with nNO levels in a large group of patients with PCD and SCD when taken together; this correlation was essentially driven by PCD subjects, and levels of nNO correlated with ciliary functional abnormalities. Furthermore, exploratory analyses have suggested that NOS3 polymorphisms may associate with particular dynein arm defects, although this is a hypothesis-generating observation that requires confirmation in a larger group of patients. The strengths of the study include the careful categorisation of a large number of patients with state-of-the-art diagnostic tests in a single centre.

There are a number of factors to consider when interpreting these data. First, the sensitivity and specificity of nNO measurements in this group appear to be different from those reported elsewhere [2]. This may relate to different techniques or equipment, and it is important for each centre to establish their own normal ranges [19]. The diagnosis of PCD was confirmed by tissue culture in all patients with higher than expected nNO levels [16], and so we are confident that the diagnosis is correct. Secondly, it was only possible to study NOS2 and NOS3 mRNA, not protein, so the possibility cannot be excluded that post-translational modification may have affected the results. Finally, the numbers were relatively small, especially for genetic analyses. This is, to some extent, inevitable in a condition as rare as PCD, but means that the genetic work in particular requires confirmation in a second cohort of patients.

Ciliate epithelial cells produce $\mathrm{NO}$, which is a regulator of $\mathrm{CBF}$ throughout NO-cyclic adenosine monophosphate (cAMP) and cyclic guanosine monophosphate (cGMP) pathways [20]. There is good evidence that cAMP and cGMP regulate mammalian $\mathrm{CBF}$ via the activation of axonemal protein kinase $\mathrm{A}$ and protein kinase $\mathrm{G}$ to phosphorylate a dynein light chain, which, in turn, possibly mediates a switch from the slow to the fast dynein duty cycle thus increasing CBF [21]. Low levels of nNO correlate with impaired mucociliary function in the upper airways [22]. Endogenous NO, principally synthesised by 
NOS2 within the respiratory epithelium of the nasal sinuses, has been demonstrated to increase the baseline CBF of airway epithelia both in vitro [23, 24] and in an animal model [25]. Here, these observations are extended by showing that there is a correlation between nNO level and ciliary motion in children with SCD or PCD. An increased CBF correlated with the level of $\mathrm{nNO}$ (fig. 1), and a higher percentage of immotile cilia correlated with a low nNO level (fig. 2). It has to be acknowledged that the scatter is wide; nonetheless, the observation has been made in a large number of patients. In human studies, instillation of the NO donor sodium nitroprusside into the noses of six volunteers was found to increase nasal mucociliary activity [26], and the administration of L-arginine was shown to improve CBF in PCD patients [27]. This body of evidence suggests that reduced nNO levels, as well as being a marker for PCD, may also contribute to its pathophysiology, and we speculate that NOS polymorphisms may be modifier genes for the PCD clinical phenotype. However, multicentric prospective studies are required to confirm this.

There is no obvious reason for the decreased nNO level in PCD patients. All three NOS isoforms have been found in airway epithelial cells $[5,28,29]$, and, here, it has been shown that NOS2 and NOS3 are found in nasal epithelial cells of PCD patients. The lower NOS2 mRNA levels in PCD compared to SCD, together with the correlation between levels of nasal $\mathrm{NO}$ and NOS2 in PCD children, suggest that impaired NOS2 function may significantly contribute to the reduced nasal NO levels in PCD subjects. However, further work is required to confirm this speculation. NO is an important host defence molecule, and is important in the response to infection and inflammation. However, relative NOS2 gene expression was reduced in PCD compared to subjects with SCD despite potential greater infectious stimuli in the former group. Thus it is tempting to speculate that, in PCD, there is a reduced potential for NOS2 expression and production, as previously hypothesised [6]. However, the burden of nasal infection and inflammation and the effect of antibiotic treatment were not measured, and so further work is clearly needed before any conclusions can be drawn as to the dose relationship between infection, inflammation and NOS2 expression in these two groups.

As suggested by the same group [6], no significant difference in NOS3 gene expression was observed between PCD and SCD patients, confirming that, in PCD, there is no generalised disorder of $\mathrm{NO}$ handling. It may be that the contribution of NOS3 to the production of $\mathrm{NO}$ in the lung periphery is important, but it does not seem to play a major rule in the nose, where NO production seems to be mainly driven by NOS2. No correlation was found in any patient group between NOS3 gene expression and nasal NO level. Nevertheless, in preliminary exploratory work, NOS3 gene expression significantly correlated with the percentage of missing central microtubules only in PCD patients and in relation to the ultrastructural localisation of NOS3 near the basal body of the microtubules of the cilia [5]. This may be a causally linked effect. However, further work is required to confirm whether or not this is a real finding, and, if so, the significance of this observation.

In the present PCD patients, NOS3 polymorphisms did not influence median nNO levels, but, in $70 \%$ of patients with $\mathrm{nNO}$ $>290 \mathrm{ppb}$, the $894 \mathrm{~T}$ allele was present. This allele (heterozygous or homozygous) inversely correlated with the most typical ultrastructural alterations, such as missing inner and outer dynein arms $(\mathrm{p}<0.001 ; \mathrm{r}=-0.563)$, and this was not the case with the wild-type GG genotype. Indeed, the prevalence of inner and outer dynein arm defects was significantly lower when the 894T allele was present (mean \pm SD $36.8 \pm 30.9$ and $7.5 \pm 16.1 \%$, respectively, in 894GG versus 894GT plus 894TT genotypes; $\mathrm{p}=0.0003)$. Clearly, there are numerous dynein arm genes, making linkage disequilibrium between NOS3 and dynein arm genes an unlikely explanation for this finding. However, it may be that in some, as yet unknown, way NOS3 polymorphisms modify the expression of dynein arm genes, leading to more or less dynein arms for a given gene defect. Again, this finding is preliminary and requires confirmation by other investigators.

Thus, $\sim 25 \%$ of total patients with the $\mathrm{T}$ allele showed higher than expected $\mathrm{nNO}$ levels. It has been observed in cystic fibrosis that NO levels were significantly higher in females with an 894T mutant allele compared with female patients homozygous for the $894 \mathrm{G}$ wild-type allele, and that chronic infection of airways with Pseudomonas aeruginosa occurred significantly less frequently when carrying an $894 \mathrm{~T}$ mutant allele as compared with wild type [11], but this is the first report of these findings in PCD. In the present study population, chronic infection with Pseudomonas aeruginosa was found in seven (31.8\%) out of 22 of the patients homozygous for the G allele and in five (19.2\%) out of 26 patients carrying the $\mathrm{T}$ variant. None of the five postpubertal females with the $\mathrm{T}$ allele was positive for Pseudomonas aeruginosa, and all of the sputum culture-positive patients were male. The apparent protective effect of the T allele against airway infection was also observed in the study in post-pubertal female patients, and we speculate that this could be related to circulating oestrogen, which activates plasma membrane-associated oestrogen receptors coupled to NOS3 [30]. However, we do not have any oestrogen measurements in these patients.

In conclusion, we suggest that $\mathrm{NO}$ is implicated in the modulation of ciliary function in PCD patients. NOS2 but not NOS3 gene expression is lower in PCD patients, and NOS3 gene expression may be correlated with loss of central microtubules. This reflects the correlation between $\mathrm{NO}$ and ciliary function, and may explain some of the clinical variability of the disease.

\section{SUPPORT STATEMENT}

This study was supported by the Fondazione Carlo Laviosa (Livorno, Italy).

\section{STATEMENT OF INTEREST}

A statement of interest for A. Bush can be found at www.erj. ersjournals.com/site/misc/statements.xhtml

\section{REFERENCES}

1 Barbato A, Frischer T, Kuehni CE, et al. Primary ciliary dyskinesia: a consensus statement on diagnostic and treatment approaches in children. Eur Respir J 2009; 34: 1264-1276.

2 Narang I, Ersu R, Wilson NM, et al. Nitric oxide in chronic airway inflammation in children: diagnostic use and pathophysiological significance. Thorax 2002; 57: 586-589.

3 Kuehni CE, Frischer T, Strippoli MP, et al. Factors influencing age at diagnosis of primary ciliary dyskinesia in European children. Eur Respir J 2010; 36: 1248-1258. 
4 Pifferi M, Bush A, Caramella D, et al. Agenesis of paranasal sinuses and nasal nitric oxide in primary ciliary dyskinesia. Eur Respir J 2011; 37: 566-571.

5 Petruson K, Stalfors J, Jacobsson KE, et al. Nitric oxide production in the sphenoidal sinus by the inducible and constitutive isozymes of nitric oxide synthase. Rhinology 2005; 43: 18-23.

6 Paraskakis E, Zihlif N, Bush A. Nitric oxide production in PCD: possible evidence for differential nitric oxide synthase function. Pediatr Pulmonol 2007; 42: 876-880.

7 Forstermann U, Boissel JP, Kleinert H. Expressional control of the "constitutive" isoforms of nitric oxide synthase (NOS I and NOS III). FASEB J 1998; 12: 773-790.

8 Marsden PA, Heng $\mathrm{HH}$, Scherer SW, et al. Structure and chromosomal localization of the human constitutive endothelial nitric oxide synthase gene. J Biol Chem 1993; 268: 17478-17488.

9 Tesauro M, Thompson WC, Rogliani $\mathrm{P}$, et al. Intracellular processing of endothelial nitric oxide synthase isoforms associated with differences in severity of cardiopulmonary diseases: cleavage of proteins with aspartate vs. glutamate at position 298. Proc Natl Acad Sci USA 2000; 97: 2832-2835.

10 Storm van's Gravesande K, Wechsler ME, Grasemann $\mathrm{H}$, et al. Association of a missense mutation in the NOS3 gene with exhaled nitric oxide levels. Am J Respir Crit Care Med 2003; 168: 228-231.

11 Grasemann H, Storm van's Gravesande $\mathrm{K}$, Buscher $\mathrm{R}$, et al. Endothelial nitric oxide synthase variants in cystic fibrosis lung disease. Am J Respir Crit Care Med 2003; 167: 390-394.

12 Baraldi E, de Jongste JC. Measurement of exhaled nitric oxide in children, 2001. Eur Respir J 2002; 20: 223-237.

13 Piacentini GL, Bodini A, Peroni D, et al. Nasal nitric oxide for early diagnosis of primary ciliary dyskinesia: practical issues in children. Respir Med 2008; 102: 541-547.

14 Pifferi M, Cangiotti AM, Ragazzo V, et al. Primary ciliary dyskinesia: diagnosis in children with inconclusive ultrastructural evaluation. Pediatr Allergy Immunol 2001; 12: 274-282.

15 Carlén B, Stenram U. Ultrastructural diagnosis in the immotile cilia syndrome. Ultrastruct Pathol 1987; 11: 653-658.

16 Pifferi M, Montemurro F, Cangiotti AM, et al. Simplified cell culture method for the diagnosis of atypical primary ciliary dyskinesia. Thorax 2009; 64: 1077-1081.

17 RANDOM.ORG. www.random.org Date last updated: April 2007. Date last accessed: June 2009
18 Pfaffl MW, Horgan GW, Dempfle L. Relative expression software tool (REST) for group-wise comparison and statistical analysis of relative expression results in real-time PCR. Nucleic Acids Res 2002; 30: e36.

19 Struben VM, Wieringa MH, Mantingh CJ, et al. Nasal NO: normal values in children age 6 through to 17 years. Eur Respir J 2005; 26: 453-457.

20 Li D, Shirakami G, Zhan X, et al. Regulation of ciliary beat frequency by the nitric oxide-cyclic guanosine monophosphate signaling pathway in rat airway epithelial cells. Am J Respir Cell Mol Biol 2000; 23: 175-181.

21 Salathe M. Regulation of mammalian ciliary beating. Anпu Rev Physiol 2007; 69: 401-422.

22 Lindberg S, Cervin A, Runer T. Low levels of nasal nitric oxide (NO) correlate to impaired mucociliary function in the upper airways. Acta Otolaryngol 1997; 117: 728-734.

23 Jain B, Rubinstein I, Robbins RA, et al. TNF-alpha and IL-1 beta upregulate nitric oxide-dependent ciliary motility in bovine airway epithelium. Am J Physiol 1995; 268: L911-L917.

24 Kim JW, Min YG, Rhee CS, et al. Regulation of mucociliary motility by nitric oxide and expression of nitric oxide synthase in the human sinus epithelial cells. Laryngoscope 2001; 111: 246-250.

25 Runer T, Cervin A, Lindberg S, et al. Nitric oxide is a regulator of mucociliary activity in the upper respiratory tract. Otolaryngol Head Neck Surg 1998; 119: 278-287.

26 Runer T, Lindberg S. Effects of nitric oxide on blood flow and mucociliary activity in the human nose. Ann Otol Rhinol Laryngol 1998; 107: 40-46.

27 Loukides S, Kharitonov S, Wodehouse T, et al. Effect of arginine on mucociliary function in primary ciliary dyskinesia. Lancet 1998; 352: $371-372$.

28 Xue C, Reynolds PR, Johns RA. Developmental expression of NOS isoforms in fetal rat lung: implications for transitional circulation and pulmonary angiogenesis. Am J Physiol 1996; 270: L88-L100.

29 Asano K, Chee CBE, Gaston B, et al. Constitutive and inducible nitric oxide synthase gene expression, regulation, and activity in human lung epithelial cells. Proc Natl Acad Sci USA 1994; 91: 10089-10093.

30 Lantin-Hermoso RL, Rosenfeld CR, Yuhanna IS, et al. Estrogen acutely stimulates nitric oxide synthase activity in fetal pulmonary artery endothelium. Am J Physiol 1997; 273: L119-L126. 